\title{
COMPARAÇÃo DA VEGETAÇÃo ARBÓREA DE CERRADÃo E DE CERRADO NA RESERVA DO PANGA, UBERLÂNDIA, MINAS GERAIS
}

\author{
Alexandre Augusto Costa ${ }^{1}$ \\ Glein Monteiro de Araújo ${ }^{2}$
}

Recebido em 29/2/2000. Aceito em 19/10/2000

\begin{abstract}
RESUMO - (Comparação da vegetação arbórea de cerradão e de cerrado na Reserva do Panga, Uberlândia, Minas Gerais). O estudo foi desenvolvido na Reserva Particular do Patrimônio Natural do Panga, localizada no Município de Uberlândia, Estado de Minas Gerais. Realizou-se, pelo método de parcelas, o levantamento florístico e a análise dos parâmetros fitossociológicos das espécies arbóreas com circunferência do tronco $\geq 15 \mathrm{~cm}$, numa faixa de $840 \mathrm{~m} \times 20 \mathrm{~m}$ (1,68ha), abrangendo comunidades vegetais contíguas de cerradão e cerrado (sentido restrito). Foram coletadas amostras compostas de solo, numa profundidade de 0 a $10 \mathrm{~cm}$, em 12 diferentes parcelas, para determinar a sua composição química. Na amostragem total foram encontrados 3.137 indivíduos distribuídos em 107 espécies e 43 famílias. Nas 25 parcelas de cerradão (1,0ha) ocorreram 93 espécies, das quais Qualea grandiflora e Vochysia tucanorum apresentaram os maiores índices de valor de importância (IVI). Em 0,68ha de cerrado, ocorreram 76 espécies e 37 famílias, sendo que as espécies de maior IVI foram Qualea grandiflora e Q. parviflora. Fabaceae, Vochysiaceae e Myrtaceae apresentaram o maior número de espécies, nas duas comunidades vegetais.
\end{abstract}

Palavras-chave - cerrado (sentido restrito), cerradão, florística, fitossociologia, Reserva do Panga

ABSTRACT - (Comparison of the arboreal vegetation of cerradão and cerrado in the Panga Reserve, Uberlândia, Minas Gerais, Brazil). The survey was carried out in the Panga Ecological Reserve, Uberlândia, Minas Gerais. A plot survey was used to analyze the floristic and phytosociology of arboreal species; with equal or more than $15 \mathrm{~cm}$ of trunk girth, in a transect of $840 \mathrm{~m} \times 20 \mathrm{~m}$ (1.68ha). The survey included plant communities of open cerrado and cerradão. Soil samples were collected, from 0 to $10 \mathrm{~cm}$ depth, in 12 different plots, to define its chemical composition. The total survey included 3,137 individuals, in 107 species and 43 families. In the cerradão 25 plots (1.0ha) presented 93 species, with Qualea grandiflora, and Vochysia tucanorum showing the highest index of importance value (IVI). In 0.68ha of cerrado we found 76 species and 37 families, with Qualea grandiflora and Qualea parviflora presenting the higher IVI index. Fabaceae Vochysiaceae and Myrtaceae showed the largest number of species in the two plant communities

Key words - Savanna, seasonal forest, floristic, phytosociology, Panga Reserve

1 Curso de Ciências Biológicas, Universidade Federal de Uberlândia

2 Universidade Federal de Uberlândia, Instituto de Biologia, C. Postal 593, CEP 38400-902, Uberlândia, MG, Brasil, e-mail: glein@ufu.br 


\section{Introdução}

O Bioma dos Cerrados caracteriza-se por apresentar uma vegetação com sinúsias de hemicriptófitos, geófitos e fanerófitos oligotróficos (Veloso 1992) que se apresentam dispostos segundo um gradiente de biomassa.

Coutinho (1978) sugere que, do ponto de vista fisionômico, os cerrados apresentam dois extremos o cerradão, fisionomia na qual predomina o componente arbóreo-arbustivo, e o campo limpo onde há predomínio do componente herbáceo-subarbustivo. As demais fisionomias encontradas - campo sujo, campo cerrado, cerrado (sentido restrito) - podem ser consideradas ecótonos entre o cerradão e o campo limpo.

As áreas marginais e disjuntas dos cerrados (Ratter et al. 1988) apresentam uma flora relativamente pobre em comparação com a área core. Porém, nessas áreas marginais podem ocorrer elementos florísticos de formações vegetais adjacentes (Rizzini 1963; Eiten 1972; Castro et al. 1999).

Alguns estudos florísticos e fitossociológicos, sobretudo do componente arbóreo e arbustivo, já foram realizados não só nas áreas centrais como nas áreas disjuntas dos Cerrados (Ratter et al. 1973; Ribeiro et al. 1985; Nascimento \& Saddi 1992; Felfili et al. 1993; Mantovani \& Martins 1993; Miranda 1993; Ratter et al. 1996; Araújo et al. 1997a; Rossi et al. 1998). Esses trabalhos mostraram que a vegetação dos Cerrados apresentam fisionomias típicas, elevada diversidade florística e espécies com ampla distribuição geográfica.

O objetivo do trabalho foi comparar os aspectos florísticos e fitossociológicos de duas comunidades vegetais contíguas uma de cerradão e outra de cerrado (sentido restrito), na Reserva do Panga, localizada no município de Uberlândia, Minas Gerais.

\section{Material e métodos}

A Reserva Particular do Patrimônio Natural (RPPN) do Panga compreende 409,5ha ao sul do município de Uberlândia à cerca de $35 \mathrm{~km}$ do centro da cidade. A Reserva está posicionada geograficamente entre as coordenadas $1^{\circ}$ $09^{\prime} 20^{\prime \prime}-19^{\circ} 11^{\prime} 10^{\prime \prime}$ de latitude sul e 48'23'20"$48^{\circ} 24^{\prime} 35^{\prime \prime}$ de longitude oeste, a uma altitude média de $800 \mathrm{~m}$, apresentando uma excelente representatividade dos diversos tipos fitofisionômicos encontrados na região do Cerrado do Brasil Central (Schiavini \& Araújo 1989).

O clima do Município de Uberlândia é do tipo Aw segundo a classificação de Köppen, com verões quentes e úmidos, e invernos frios e secos (Rosa et al. 1991). Na região, são encontrados principalmente Latossolos Vermelho-Escuros, variando de moderados a fortemente ácidos (Embrapa 1982).

A área de estudo, na Reserva do Panga, constituiu-se numa faixa de $20 \mathrm{~m}$ x $840 \mathrm{~m}$ $(1,68 \mathrm{ha})$, ao longo das fitofisionomias de cerradão e cerrado (sentido restrito), definidos a partir da densidade, altura e cobertura de copa da vegetação arbórea.

A faixa (transeção) adotada foi subdivida em 42 parcelas de $20 \mathrm{~m}$ x 20m, nas quais foram amostrados todos os indivíduos vivos das espécies arbóreas, com perímetro do tronco à altura do peito ou abaixo do primeiro galho vivo, $\geq$ $15 \mathrm{~cm}$. Dos indivíduos que apresentavam troncos múltiplos, foram incluídos na amostragem apenas aqueles nos quais pelo menos um dos troncos atingiu o perímetro mínimo estabelecido.

Das 42 parcelas analisadas, $25(59,5 \%)$ encontravam-se na área de cerradão e 17 (40,5\%) na fisionomia de cerrado. Além da altura total, também determinou-se, utilizando uma vara com escala métrica, a altura até o primeiro galho vivo, de todas as árvores amostradas.

A partir das variáveis obtidas foram calculados os seguintes parâmetros fitossociológicos: densidade relativa (DR), dominância relativa (DoR), frequiência relativa (FR), índice de valor de importância (IVI), índice de diversidade de Shannon (H'), índice de uniformidade (equabilidade) e índice de similaridade florística 
de acordo com Sorensen, usando o programa FITOPAC 1 (Sheperd 1994).

Para caracterizar a composição química do solo, coletaram-se amostras compostas, numa profundidade de 0 a $10 \mathrm{~cm}$, em sete parcelas do cerradão e cinco do cerrado. As amostras foram analisadas quanto ao $\mathrm{pH}$ e às concentrações de P, K, Ca, Mg, Al e matéria orgânica. Tais análises foram realizadas, de acordo com métodos da Embrapa (1982), pelo Laboratório de Análises de Solos do Departamento de Agronomia da Universidade Federal de Uberlândia.

\section{Resultados e discussão}

Os solos sob as comunidades vegetais de cerradão e cerrado (sentido restrito) da Reserva do Panga (Tab. 1) apresentaram baixas concentração de nutrientes disponíveis que os caracterizam, de acordo com Lopes \& Cox (1977), como ácidos e distróficos. Valores semelhantes foram encontrados por Lopes \& Cox (1977), Ratter et al. (1977) e Goodland (1979) em solos sob cerrado e cerradão no Brasil Central.

As principais características fitossociológicas das comunidades vegetais estudadas encontram-se na Tab. 2. Em toda a área amostrada (1,68ha) foram encontrados $3.137 \mathrm{in}$ divíduos arbóreos distribuídos em 107 espécies e 43 famílias. O cerradão, comunidade vegetal de porte florestal, teve maiores valores para todas as características exceto para o índice de diversidade de Shannon para espécies.

A riqueza de espécies obtida, na área total, assemelha-se a outros levantamentos do com- ponente arbóreo e arbustivo de comunidades vegetais dos cerrados. Ratter et al. (1973), estudando áreas de cerrado e cerradão no nordeste do Mato Grosso, citam 156 espécies arbustivas de grande porte e arbóreas. Ribeiro et al. (1985) amostraram 91 espécies lenhosas em Planaltina, Distrito Federal. Felfili et al. (1993) encontraram 139 espécies nas áreas de cerrado (sentido restrito) da Chapada Pratinha. Deve-se notar que foram amostrados apenas os indivíduos com perímetro igual ou superior a $15 \mathrm{~cm}$, o que restringe o espaço amostral ao componente arbóreo da vegetação, bem mais representativo na fisionomia de cerradão.

A similaridade florística de espécies entre o cerradão e o cerrado, baseada no índice de Sorensen, foi de 73,4\%. Este valor de similaridade pode ser considerado alto para duas fisionomias distintas, se comparado a outros trabalhos em áreas de Cerrado. A determinação da elevada similaridade florística entre as duas comunidades vegetais do Panga pode ser devida ao fato de que o cerradão e cerrado constituem áreas contíguas, permitindo que espécies com alto potencial de dispersão possam ocupar mais de uma fisionomia. $\mathrm{O}$ índice de similaridade entre cerradão e cerrado estudados por Ribeiro et al. (1985) no Centro de Pesquisa Agropecuária do cerrado em Planaltina, Distrito Federal, foi de $35 \%$. Nas seis áreas de cerrado (sentido restrito) estudadas por Felfili et al. (1993) na Chapada Pratinha, situada em Minas Gerais, Goiás e Distrito Federal, a similaridade esteve entre 51 e $77 \%$, sendo o maior valor entre o Par-

Tabela 1. Características do solo sob as comunidades vegetais de cerradão e de cerrado na Reserva do Panga, Uberlândia, Minas Gerais. Desvio-padrão entre parênteses.

\begin{tabular}{|c|c|c|c|c|c|c|c|}
\hline $\begin{array}{c}\text { Comunidade } \\
\text { vegetal }\end{array}$ & $\mathrm{pH}$ & $\begin{array}{c}\mathrm{P} \\
\left(\mathrm{mg} / \mathrm{dm}^{3}\right)\end{array}$ & $\begin{array}{c}\mathrm{K} \\
\left(\mathrm{mg} / \mathrm{dm}^{3}\right)\end{array}$ & $\begin{array}{c}\mathrm{Ca} \\
\left(\mathrm{cmol} . / \mathrm{dm}^{3}\right)\end{array}$ & $\begin{array}{c}\mathrm{Mg} \\
\left(\mathrm{cmol} . / \mathrm{dm}^{3}\right)\end{array}$ & $\begin{array}{c}\mathrm{Al} \\
\left(\mathrm{cmol} . / \mathrm{dm}^{3}\right)\end{array}$ & $\begin{array}{l}\text { Matéria } \\
\text { Orgânica } \\
\text { (dag/kg) }\end{array}$ \\
\hline $\begin{array}{l}\text { Cerradão } \\
(\mathrm{n}=7)\end{array}$ & $\begin{array}{c}4,6 \\
( \pm 0,21)\end{array}$ & $\begin{array}{c}2,86 \\
( \pm 1,67)\end{array}$ & $\begin{array}{c}27,70 \\
( \pm 4,56)\end{array}$ & $\begin{array}{c}0,17 \\
( \pm 0,08)\end{array}$ & $\begin{array}{c}0,01 \\
( \pm 0,04)\end{array}$ & $\begin{array}{c}1,13 \\
( \pm 0,24)\end{array}$ & $\begin{array}{c}2,24 \\
( \pm 0,40)\end{array}$ \\
\hline $\begin{array}{l}\text { Cerrado } \\
(n=5)\end{array}$ & $\begin{array}{c}4,9 \\
( \pm 0,09)\end{array}$ & $\begin{array}{c}1,64 \\
( \pm 0,36)\end{array}$ & $\begin{array}{r}29,00 \\
( \pm 4,64)\end{array}$ & $\begin{array}{c}0,28 \\
( \pm 0,29)\end{array}$ & $\begin{array}{c}0,00 \\
( \pm 0,00)\end{array}$ & $\begin{array}{l}1,00 \\
( \pm 0,20)\end{array}$ & $\begin{array}{l}2,14 \\
( \pm 0,90)\end{array}$ \\
\hline
\end{tabular}


Tabela 2. Características fitossociológicas gerais das comunidades vegetais arbóreas de cerradão e cerrado na Reserva do Panga, Uberlândia, Minas Gerais.

\begin{tabular}{lccc}
\hline \multicolumn{1}{c}{ Características } & Cerradão & Cerrado & Total \\
\hline Área amostrada (ha) & 1,00 & 0,68 & 1,68 \\
Número de espécies & 93 & 76 & 107 \\
Número de famílias & 41 & 37 & 43 \\
Espécies exclusivas & 31 & 14 & - \\
Famílias exclusivas & 6 & 2 & - \\
Densidade & 2.071 & 1.066 & 3.137 \\
Área basal (m²/ha) & 17,06 & 9,63 & 26,69 \\
Altura média (m) & $5,96 \pm 2,62^{1}$ & $3,94 \pm 1,70^{1}$ & $5,27 \pm 2,54^{1}$ \\
Altura média (m) do primeiro galho vivo & $2,57 \pm 1,47^{1}$ & $1,52 \pm 0,85^{1}$ & - \\
Equabilidade (J) & 0,78 & 0,84 & 0,82 \\
Índice de Shannon (H') para espécies (nats/indivíduo) & 3,54 & 3,63 & 3,84 \\
\hline
\end{tabular}

${ }^{1}=$ média \pm desvio padrão

que Nacional de Brasília e a Área de Proteção Ambiental Gama-Cabeça de Veado. Miranda \& Carneiro-Filho (1994), analisando a similaridade entre gêneros presentes em sete savanas de terra-firme da Amazônia brasileira, áreas disjuntas do Cerrado brasileiro, obteve índices entre 30 e $40 \%$.

De acordo com Rizzini (1979), muitas espécies que ocorrem no cerradão são também encontradas em matas mesófilas semidecíduas. No cerradão da Reserva do Panga, 38,7\% das espécies encontradas ocorrem em matas mesófilas semidecíduas do Triângulo Mineiro (Tab. 3) como pode ser constatado nos trabalhos de Araújo \& Haridasan (1997) e Araújo et al. (1997b). As espécies comuns ao cerradão e às matas mesófilas semidecíduas, em geral não apresentam valores de importância similares nas duas comunidades. Coussarea hydrangeaefolia, Matayba guianensis, Ocotea puchella, Rudgea viburnoides e Vochysia tucanorum, que ocorreram entre as dez de maior valor de importância no cerradão não apresentam essa característica nas matas estudadas no Triângulo Mineiro. Por outro lado Alibertia sessilis, Copaifera langsdorffii, Ocotea corymbosa, Tapirira guianensis, Virola sebifera, e outras, possuem maiores valores de importâncias nas matas. Enfim, as espécies de matas que são encontradas no cerradão parecem ocorrer em seu limite de distribuição, caracterizando o cerradão como uma possível área de transição entre as matas e o cerrado.

A densidade arbórea apresentada pelo cerradão foi cerca de 1,32 vezes maior que a do cerrado. Essa diferença é inferior à determinada por Ribeiro et al. (1985) para espécies arbóreas na região de Planaltina, no Distrito Federal, calculada 2,45 vezes maior no cerradão. As diferenças de densidade entre essas fitocenoses não são tão discrepantes, haja vista que o cerrado é a formação savânica fisionomicamente mais próxima ao cerradão no conceito floresta-ecótonocampo de Coutinho (1978).

A análise da altura dos galhos vivos mais próximos ao solo, de todos os indivíduos amostrados (Fig. 1), permite constatar que na fisionomia de cerradão são mais freqüentes as alturas entre 1,5 e $2,0 \mathrm{~m}$, enquanto que no cerra-

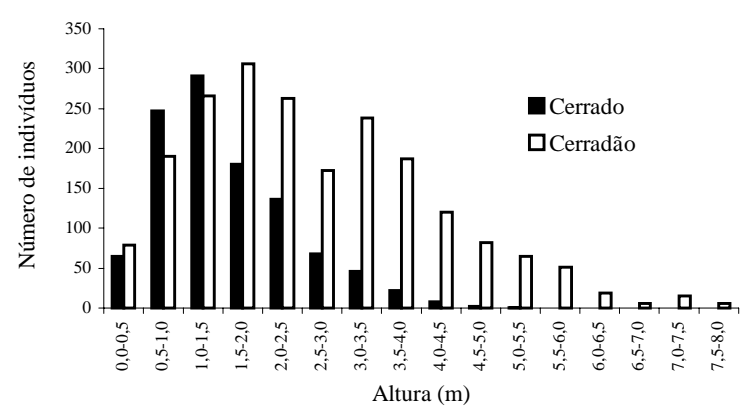

Figura 1. Distribuição de freqüência, da altura do primeiro galho vivo, nas espécies arbóreas de cerradão e cerrado na Reserva do Panga, Uberlândia, Minas Gerais. $n=2071$ indivíduos para cerradão e 1.066 para cerrado. 
Tabela 3. Características fitossociológicas das espécies amostradas nas duas comunidades vegetais da Reserva do Panga Uberlândia, MG, em ordem de IVI para cerradão. $\mathrm{N}=$ Número de indivíduos amostrados, $\mathrm{CR}=$ Cerradão, $\mathrm{CE}=$ cerrado. * = espécies que ocorrem em matas mesófilas semidecíduas no Triângulo Mineiro (Araújo et al. 1997b, Araújo \& Haridasan 1997).

\begin{tabular}{|c|c|c|c|c|c|}
\hline \multirow[b]{2}{*}{ Espécies } & \multirow[b]{2}{*}{ Famílias } & \multicolumn{2}{|c|}{$\mathrm{N}$} & \multicolumn{2}{|c|}{ IVI } \\
\hline & & $\mathrm{CR}$ & $\mathrm{CE}$ & $\mathrm{CR}$ & $\mathrm{CE}$ \\
\hline Qualea grandiflora Mart. & Vochysiaceae & 279 & 132 & 35,06 & 28,73 \\
\hline *Vochysia tucanorum Mart. & Vochysiaceae & 269 & 0 & 31,95 & 0 \\
\hline *Matayba guianensis Aubl. & Sapindaceae & 144 & 1 & 14,27 & 0,36 \\
\hline Xylopia aromatica (Lam.) Mart. & Annonaceae & 87 & 15 & 10,0 & 4,06 \\
\hline *Coussarea hydrangeaefolia Benth. \& Hook. & Rubiaceae & 82 & 2 & 9,21 & 0,70 \\
\hline Miconia albicans $($ Sw.) Triana & Melastomataceae & 89 & 27 & 9,19 & 6,06 \\
\hline *Rudgea viburnoides (Cham.) Benth. & Rubiaceae & 79 & 10 & 8,90 & 2,38 \\
\hline Symplocos $\mathrm{sp} .1$ & Symplocaceae & 77 & 2 & 8,49 & 0,45 \\
\hline *Ocotea pulchella Mart. & Lauraceae & 76 & 0 & 8,38 & 0 \\
\hline Bowdichia virgilioides H. B. \& K. & Fabaceae & 27 & 14 & 7,56 & 7,25 \\
\hline Acosmium dasycarpum (Vog.) Yakovlev & Fabaceae & 38 & 23 & 6,78 & 7,54 \\
\hline *Virola sebifera Aubl. & Myristicaceae & 51 & 1 & 6,35 & 0,33 \\
\hline *Tapirira guianensis Aubl. & Anacardiaceae & 35 & 0 & 6,30 & 0 \\
\hline *Ocotea corymbosa (Miers.) Mez. & Lauraceae & 22 & 0 & 6,08 & 0 \\
\hline Qualea parviflora Mart. & Vochysiaceae & 41 & 91 & 5,97 & 27,07 \\
\hline Guapira noxia (Netto) Lundell & Nyctaginaceae & 30 & 19 & 5,92 & 5,53 \\
\hline Cardiopetalum calophyllum Schlecht & Annonaceae & 53 & 1 & 5,74 & 0,35 \\
\hline *Myrcia rostrata DC. & Myrtaceae & 37 & 18 & 5,67 & 4,13 \\
\hline Vatairea macrocarpa (Benth.) Ducke & Fabaceae & 14 & 3 & 5,25 & 2,12 \\
\hline Caryocar brasiliense Camb. & Caryocaraceae & 24 & 49 & 5,15 & 18,76 \\
\hline *Myrcia tomentosa $\mathrm{DC}$ & Myrtaceae & 29 & 0 & 4,11 & 0 \\
\hline *Copaifera langsdorffii Desf. & Caesalpiniaceae & 21 & 0 & 4,04 & 0 \\
\hline *Pera glabrata (Schott) Baill. & Euphorbiaceae & 22 & 0 & 4,02 & 0 \\
\hline Roupala montana Aubl. & Proteaceae & 20 & 20 & 3,94 & 5,10 \\
\hline Dimorphandra mollis Benth. & Caesalpiniaceae & 25 & 4 & 3,83 & 1,28 \\
\hline Qualea multiflora Mart. & Vochysiaceae & 21 & 12 & 3,55 & 3,21 \\
\hline Myrcia variabilis Mart. ex DC. & Myrtaceae & 18 & 22 & 2,99 & 4,69 \\
\hline Licania humilis Cham. ex Schlecht. & Chrysobalanaceae & 14 & 25 & 2,96 & 7,05 \\
\hline Guapira graciliflora (Mart. ex Schmidt) Lundell & Nyctaginaceae & 16 & 4 & 2,90 & 1,19 \\
\hline Symplocos sp. 2 & Symplocaceae & 24 & 15 & 2,81 & 2,95 \\
\hline Couepia grandiflora (Mart. \& Zucc.) Bent. \& Hook. f. & Chrysobalanaceae & 10 & 9 & 2,47 & 3,82 \\
\hline Salvertia convallariaeodora A. St.-Hil. & Vochysiaceae & 8 & 5 & 2,37 & 1,56 \\
\hline * Sclerolobium paniculatum Vog. & Caesalpiniaceae & 13 & 0 & 2,35 & 0 \\
\hline *Rapanea umbellata (Mart.) Mez. & Myrsinaceae & 15 & 0 & 2,23 & 0 \\
\hline Pouteria ramiflora (Mart.) Radlk. & Sapotaceae & 6 & 21 & 2,17 & 10,32 \\
\hline *Alibertia sessilis (Vell.) K.Schum. & Rubiaceae & 14 & 0 & 2,02 & 0 \\
\hline Byrsonima crassa Niedensu & Malpighiaceae & 13 & 59 & 2,01 & 12,7 \\
\hline *Qualea dichotoma (Mart.) Warm. & Vochysiaceae & 13 & 0 & 2,00 & 0 \\
\hline Andira paniculata Benth. & Fabaceae & 10 & 9 & 1,91 & 2,63 \\
\hline *Platypodium elegans Vog. & Fabaceae & 6 & 0 & 1,86 & 0 \\
\hline *Terminalia brasiliensis Raddi & Combretaceae & 11 & 0 & 1,85 & 0 \\
\hline Didymopanax macrocarpum (Cham. \& Schlecht.) Seem & Araliaceae & 7 & 11 & 1,70 & 2,81 \\
\hline *Hirtella gracilipes (Hook. f.) Prance & Chrysobalanaceae & 12 & 0 & 1,62 & 0 \\
\hline Brosimum gaudichaudii Tréc. & Moraceae & 8 & 7 & 1,56 & 2,41 \\
\hline Machaerium opacum Vog. & Fabaceae & 7 & 2 & 1,50 & 0,71 \\
\hline Austroplenckia populnea (Reiss.) Lundell & Celastraceae & 6 & 3 & 1,44 & 1,09 \\
\hline Plathymenia reticulata Benth. & Mimosaceae & 7 & 3 & 1,44 & 1,18 \\
\hline
\end{tabular}


Tabela 2. (Continuação)

\begin{tabular}{|c|c|c|c|c|c|}
\hline \multirow[b]{2}{*}{ Espécies } & \multirow[b]{2}{*}{ Famílias } & \multicolumn{2}{|c|}{$\mathrm{N}$} & \multicolumn{2}{|c|}{ IVI } \\
\hline & & $\overline{\mathrm{CR}}$ & $\mathrm{CE}$ & $\mathrm{CR}$ & CE \\
\hline Eriotheca gracilipes (K. Schum.) A. Robyns & Bombacaceae & 8 & 6 & 1,40 & 2,45 \\
\hline Tabebuia ochracea (Cham.) Standley & Bignoniaceae & 5 & 6 & 1,37 & 1,93 \\
\hline Annona crassiflora Mart. & Annonaceae & 7 & 24 & 1,34 & 7,81 \\
\hline Kielmeyera coriacea (Spreng.) Mart. & Clusiaceae & 9 & 71 & 1,34 & 16,45 \\
\hline Ouratea hexasperma (A. St. Hil.) Baill. & Ochnaceae & 8 & 6 & 1,34 & 2,02 \\
\hline Pouteria torta (Mart.) Radlk. & Sapotaceae & 3 & 2 & 1,25 & 0,99 \\
\hline Dalbergia miscolobium Benth. & Fabaceae & 4 & 4 & 1,21 & 1,14 \\
\hline Vochysia rufa Mart. & Vochysiaceae & 6 & 35 & 1,20 & 9,43 \\
\hline *Maprounea guianensis (Aubl.) M. Arg. & Euphorbiaceae & 5 & 0 & 0,94 & 0 \\
\hline *Hirtella glandulosa Spreng. & Chrysobalanaceae & 6 & 0 & 1,13 & 0 \\
\hline Lafoensia pacari St.Hil. & Lythraceae & 6 & 19 & 1,12 & 5,22 \\
\hline Acosmium subelegans (Mohl.) Yakovlev & Fabaceae & 4 & 1 & 1,10 & 0,36 \\
\hline Stryphnodendron polyphyllum Mart. & Mimosaceae & 4 & 36 & 0,86 & 8,94 \\
\hline Psidium firmum Berg & Myrtaceae & 4 & 1 & 0,85 & 0,35 \\
\hline Byrsonima coccolobifolia $\mathrm{H}$. B. \& K. & Malpighiaceae & 4 & 18 & 0,81 & 5,63 \\
\hline Rapanea guianensis Aubl. & Myrsinaceae & 6 & 3 & 0,81 & 0,87 \\
\hline *Apuleia leiocarpa (Vog.) Macbride & Caesalpiniaceae & 2 & 0 & 0,72 & 0 \\
\hline *Coccoloba mollis Casar & Polygonaceae & 3 & 0 & 0,67 & 0 \\
\hline Tabebuia aurea (Manso) Benth. \& Hook. f. ex. S. Moore & Bignoniaceae & 2 & 2 & 0,65 & 0,76 \\
\hline Byrsonima basiloba A. Juss. & Malpighiaceae & 3 & 5 & 0,65 & 2,18 \\
\hline *Qualea jundiahy Warm. & Vochysiaceae & 4 & 0 & 0,63 & 0 \\
\hline *Ficus enormis (Mart. ex Miq.) Miq. & Moraceae & 1 & 0 & 0,59 & 0 \\
\hline Machaerium acutifolium Mart. ex Benth. & Fabaceae & 3 & 2 & 0,55 & 0,49 \\
\hline Sclerolobium aureum (Tul.) Benth. & Caesalpiniaceae & 2 & 1 & 0,54 & 0,35 \\
\hline Davilla elliptica A.St.-Hil. & Dilleniaceae & 3 & 31 & 0,52 & 6,89 \\
\hline Strychnos pseudoquina A.St.-Hil. & Loganiaceae & 2 & 2 & 0,51 & 0,83 \\
\hline Hymenaea stigonocarpa Mart .ex Hayne & Caesalpiniaceae & 2 & 1 & 0,50 & 0,62 \\
\hline *Faramea cyanea Muell. Arg. & Rubiaceae & 3 & 0 & 0,47 & 0 \\
\hline *Alibertia macrophylla K. Schum. & Rubiaceae & 2 & 0 & 0,42 & 0 \\
\hline *Ocotea sp. & Lauraceae & 3 & 0 & 0,40 & 0 \\
\hline Psidium pohlianum Berg & Myrtaceae & 1 & 1 & 0,40 & 0,34 \\
\hline Cybistax antisiphilitica (Mart.) Mart. & Bignoniaceae & 1 & 0 & 0,33 & 0 \\
\hline Styrax ferrugineus Ness \& Mart. & Styracaceae & 2 & 17 & 0,28 & 5,11 \\
\hline *Tabebuia serratifolia (Vahl.) Nicholson & Bignoniaceae & 1 & 0 & 0,25 & 0 \\
\hline *Aspidosperma subincanum Mart. & Apocynaceae & 1 & 0 & 0,22 & 0 \\
\hline *Protium heptaphyllum (Aubl.) March. & Burseraceae & 1 & 0 & 0,21 & 0 \\
\hline Connarus suberosus Planch. & Connaraceae & 1 & 3 & 0,21 & 1,03 \\
\hline Erythroxylum suberosum A. St.-Hil. & Erythroxylaceae & 1 & 21 & 0,21 & 5,05 \\
\hline Eugenia punicifolia (H. B. \& K.) DC. & Myrtaceae & 1 & 3 & 0,20 & 1,05 \\
\hline *Ouratea castaneaefolia (DC.) Engl. & Ochnaceae & 1 & 0 & 0,20 & 0 \\
\hline *Casearia sylvestris $\mathrm{Sw}$. & Flacourtiaceae & 1 & 0 & 0,21 & 0 \\
\hline *Luehea grandiflora Mart. \& Zucc. & Tiliaceae & 1 & 0 & 0,21 & 0 \\
\hline Hancornia speciosa Gomez & Apocynaceae & 1 & 3 & 0,20 & 1,01 \\
\hline *Agonandra brasiliensis Benth. \& Hook. f. & Opiliaceae & 1 & 4 & 0,20 & 1,25 \\
\hline Tocoyena formosa (Cham. \& Schlecht) K. Schum. & Rubiaceae & 1 & 3 & 0,20 & 1,05 \\
\hline *Pouteria gardinerii (Mart. \& Miq.) Baehni & Sapotaceae & 1 & 0 & 0,20 & 0 \\
\hline Eremanthus glomerulatus Less. & Asteraceae & 0 & 6 & 0 & 1,69 \\
\hline Piptocarpha rotundifolia (Less.) Baker & Asteraceae & 0 & 11 & 0 & 3,88 \\
\hline Tabebuia sp. & Bignoniaceae & 0 & 1 & 0 & 0,48 \\
\hline Kielmeyera rubriflora Camb. & Clusiaceae & 0 & 8 & 0 & 3,57 \\
\hline
\end{tabular}


Tabela 2. (Continuação)

\begin{tabular}{|c|c|c|c|c|c|}
\hline \multirow[b]{2}{*}{ Espécies } & \multirow[b]{2}{*}{ Famílias } & \multicolumn{2}{|c|}{$\mathrm{N}$} & \multicolumn{2}{|c|}{ IVI } \\
\hline & & $\overline{\mathrm{CR}}$ & $\mathrm{CE}$ & $\overline{\mathrm{CR}}$ & $\mathrm{CE}$ \\
\hline Diospyros burchellii DC. & Ebenaceae & 0 & 5 & 0 & 1,25 \\
\hline *Diospyros hispida A. DC. & Ebenaceae & 0 & 1 & 0 & 0,34 \\
\hline Erythroxylum deciduum A. St.-Hil. & Erythroxylaceae & 0 & 11 & 0 & 2,90 \\
\hline Erythroxylum tortuosum Mart. & Erythroxylaceae & 0 & 14 & 0 & 3,56 \\
\hline Byrsonima verbascifolia Juss. & Malpighiaceae & 0 & 1 & 0 & 0,43 \\
\hline Enterolobium gummiferum (Mart.) Macbride & Mimosaceae & 0 & 2 & 0 & 0,57 \\
\hline Stryphnodendron adstringens (Mart.) Coville & Mimosaceae & 0 & 2 & 0 & 0,75 \\
\hline Neea theifera Oerst. & Nyctaginaceae & 0 & 2 & 0 & 0,67 \\
\hline Palicourea rigida Kunth & Rubiaceae & 0 & 10 & 0 & 2,54 \\
\hline Vochysia cinnamomea Pohl & Vochysiaceae & 0 & 22 & 0 & 5,28 \\
\hline
\end{tabular}

do (sentido restrito) eles estão predominantemente entre 1,0 e 1,5m do solo. É possível que a redução na incidência solar ocasionada pela maior densidade da vegetação arbórea no cerradão possa aumentar a competição da copa por luz, favorecendo os galhos mais altos em detrimento aos mais baixos. No cerrado (sentido restrito) a densidade da vegetação arbórea é relativamente menor e permite maior penetração de luz, viabilizando a existência de galhos vivos mais baixos.

Nas duas áreas amostradas foram encontradas 43 famílias (Tab. 3), das quais seis foram exclusivas do cerradão: Burseraceae, Combretaceae, Euphorbiaceae, Flacourtiaceae, Lauraceae e Polygonaceae enquanto apenas duas ocorreram restritamente no cerrado, Asteraceae e Ebenaceae. O cerradão apresentou 41 famílias, sendo que as dez mais ricas compreenderam $54,8 \%$ das espécies amostradas. No cerrado, ocorreram 37 famílias e as mais representativas compreenderam 55,3\% das espécies (Fig. 2).

Embora Fabaceae seja a família com maior número de espécies em ambas as fisionomias, Vochysiaceae foi a que apresentou maior densidade, com 641 indivíduos no cerradão e 297 no cerrado. Fabaceae, Vochysiaceae e Myrtaceae foram as mais representativas nas duas fitocenoses, o que demonstra a presença destas famílias na configuração da vegetação arbórea

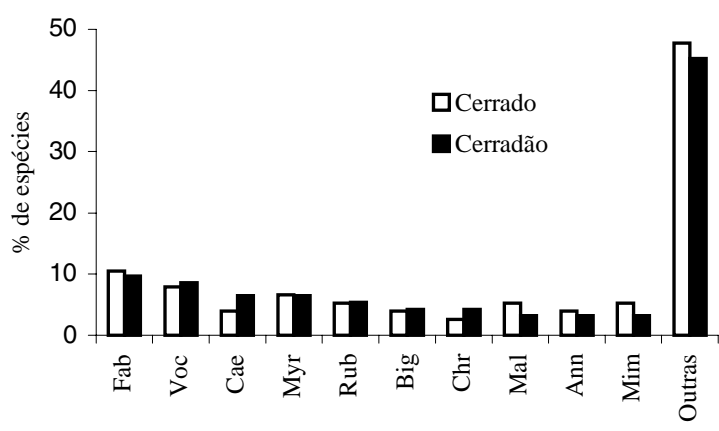

Figura 2. Famílias com maior percentual de espécies no cerradão e seus respectivos valores no cerrado da Reserva do Panga, Uberlândia, Minas Gerais. Fab = Fabaceae, Voc = Vochysiaceae, $\mathrm{Cae}=$ Caesalpiniaceae, $\mathrm{Myr}=$ Myrtaceae, $\mathrm{Rub}$ = Rubiaceae, $\mathrm{Big}=$ Bignoniaceae, $\mathrm{Chr}=$ Chrysobalanaceae, Mal = Malpighiaceae, Ann = Annonaceae e Mim = Mimosaceae.

nessas comunidades. Em levantamento da estrutura comunitária de áreas de cerrados residuais no município de Uberlândia, realizado por Araújo et al. (1997a), Fabaceae e Vochysiaceae tiveram maior densidade e freqüência de espécies, porém o maior número de espécies foi registrado para Myrtaceae.

$\mathrm{Na}$ área de cerradão da Reserva do Panga, as dez espécies mais importantes (Fig. 3) corresponderam a $47,67 \%$ do IVI total. Os maiores valores de importância de Qualea grandiflora de Vochysia tucanorum ocorreram devido aos elevados percentuais de dominância e densidade. As duas espécies são árvores cujas copas, em geral, ocupam o dossel superior e os 
seus maiores valores de dominância foram proporcionados, principalmente, pelo diâmetro médio do tronco relativamente maior que as demais. Exceto Bowdichia virgilioides, as demais espécies mais importantes, ocupam o estrato intermediário e inferior e tiveram maiores valores de IVI devido a densidade e frequiência.

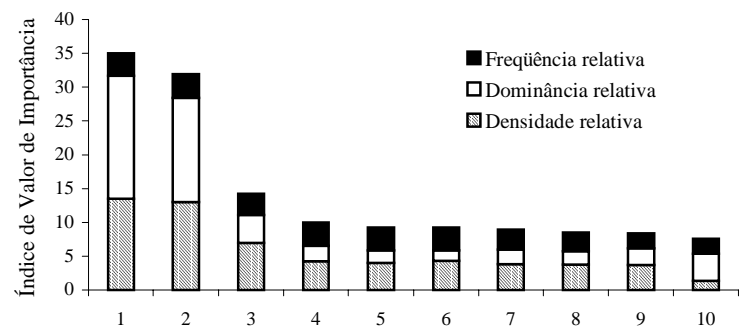

Figura 3. Parâmetros fitossociológicos das espécies de maior IVI no cerradão da Reserva do Panga. Uberlândia, Minas Gerais. 1 = Qualea grandiflora, 2 = Vochysia tucanorum, 3 = Matayba guianensis, $4=$ Xylopia aromatica, $5=$ Coussarea hydrangeaefolia, 6 = Miconia albicans, $7=$ Rudgea viburnoides, $8=$ Symplocos sp. 1, 9= Ocotea pulchella 10 = Bowdichia virgilioides.

Ratter et al. (1977) advertem que a estrutura fitossociológica do cerradão pode variar em decorrência do tipo de solo sob a comunidade vegetal. Segundo esses autores, a presença de Magonia pubescens e Callisthene fasciculata com elevada densidade e frequiência caracterizariam o cerradão em solo mesotrófico. Em cerradões de solos distróficos haveria a predominância de Hirtella glandulosa e Emmotum nitens, enquanto Qualea parviflora e Qualea grandiflora ocorreriam nos cerradões não diferenciados. Apesar de Emmotum nitens não ter sido encontrada e Hirtella glandulosa apresentar baixa densidade ficou registrado que $Q$. grandiflora e Vochysia tucanorum são espécies que se destacam na fisionomia da comunidade vegetal de cerradão, em solo distrófico, na Reserva do Panga.

A presença de Vochysia tucanorumé geralmente constatada em levantamentos florísticos de matas ciliares (Carvalho et al. 1995; Vilela et al. 1995) e matas mesófilas semidecíduas (Oliveira-Filho \& Machado 1993; Pedralli et al.
1997). Porém, os estudos fitossociológicos em matas mesófilas (Rodrigues et al. 1989; Grombone et al. 1990; Araújo \& Haridasan 1997; Araújo et al. 1997b) mostram que essa espécie apresenta baixos valores de IVI nessa formação vegetal. V.tucanorum, juntamente com Myrcia rostrata, Ocotea corymbosa, $O$. pulchella e Tapirira guianensis, são consideradas espécies secundárias iniciais em matas mesófilas semidecíduas no Estado de São Paulo (Gandolfi et al. 1995).

No cerrado da Reserva do Panga as dez espécies mais importantes tiveram 49,3\% do IVI total (Fig. 4). Os percentuais de dominância influenciaram na composição do IVI, principalmente nas quatro espécie mais importantes (Qualea grandiflora, Q. parviflora, Caryocar brasilense e Kielmeyera coriacea). As três primeiras espécies marcam a fisionomia do cerrado com árvores altas, com galhos e troncos tortuosos e suberosos. Qualea parviflora, além de atingir o segundo maior IVI, foi a única espécie que ocorreu em todas as parcelas da fisionomia do cerrado, com frequiência absoluta de $100 \%$. Esta espécie é considerada por Goodland (1979) e Ribeiro et al. (1985) uma das mais importantes nas fisionomias dos cerrados do Brasil Central. Das 76 espécies presentes nesta comunidade, 38\% apresentaram menos de três indivíduos na amostragem. Algumas delas como Hancornia speciosa, Hymenaea stigonocarpa,

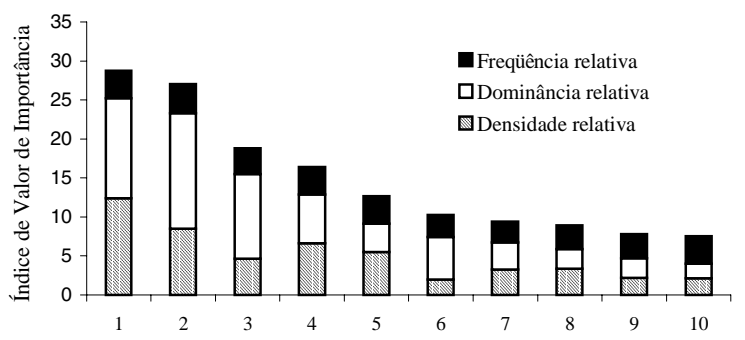

Figura 4. Parâmetros fitossociológicos obtidos nas espécies de maior IVI no cerrado da Reserva do Panga. Uberlândia, Minas Gerais. 1 = Qualea grandiflora, 2 = Qualea parviflora, $3=$ Caryocar brasiliense, $4=$ Kielmeyera coriacea, $5=$ Byrsonima crassa, $6=$ Pouteria ramiflora, $7=$ Vochysia rufa, $8=$ Stryphnodendron polyphyllum, $9=$ Annona crassiflora e $10=$ Acosmium dasycarpum. 
Machaerium acutifolium, Sclerolobium aureum, Strycnos pseudoquina, Machaerium opacum, Plathymenia reticulata e Tabebuia aurea, de acordo com Araújo et al. (1997a), são freqüentes em cerrados dos Município de Uberlândia.

Diferentemente do cerradão, a área de cerrado da Reserva do Panga apresenta uma estrutura fitossociológica bem similar à encontrada no Distrito Federal. Das dez espécies mais importantes, cinco estiveram entre as de maior IVI no cerrado estudado em Planaltina por Ribeiro et al. (1985). Qualea grandiflora Q Q. parviflora são espécies importantes e contribuem com um grande percentual da biomassa arbórea nos cerrados brasileiros (Ribeiro et al. 1985; Ratter et al. 1996). Além da acentuada dominância Qualea grandiflora, possui ampla distribuição na região do cerrado, ocorrendo em $88 \%$ dos cerrados listados por Ratter et al. (1996). A presença dessa espécie com o maior IVI nas duas comunidade vegetais da Reserva do Panga reforça o que foi proposto por Araújo \& Haridasan (1988) e Araújo et al. (1997a) de que $Q$. grandiflora possa apresentar ampla vantagem adaptativa em relação às demais do cerrado.

Com exceção de $Q$. grandiflora, que esteve presente nas duas áreas com maior IVI, houve diferenças entre as demais espécies que compõem as duas comunidades vegetais estudadas. Vochysia tucanorum e Ocotea pulchella apresentaram elevado IVI e ocorreram exclusivamente no cerradão, podendo, portanto, serem consideradas espécies típicas desta fisionomia na Reserva do Panga. Matayba guianensis, Coussarea hydrangeaefolia, Virola sebifera e Cardiopetallum callophylum apresentaram altos índices de valor de importância no cerradão, mas não foram exclusivas desse ambiente, ocorrendo também no cerrado, porém, com baixas densidades e frequiências. Matayba guianensis, Miconia albicans, Myrcia rostrata e Xylopia aromatica são espécies que ocorrem com elevada densidade em cerrados alterados do município de Uberlândia (Araújo et al. 1997a), o que pode indicar que o cerradão e o cerrado da
Reserva do Panga possam estar se recuperando do impacto de eventuais ações antrópicas que ocorreram no passado.

\section{Referências bibliográficas}

Araújo, G. M. \& Haridasan, M. A. 1988. A comparision of the nutritional status of two forest communities on mesotrophic and dystrophic soils in Central Brazil. Soil Science and Plant Analysis 19: 10757089.

Araújo, G. M. \& Haridasan, M. 1997. Estrutura fitossociológica de duas matas mesófilas semidecíduas, em Uberlândia, Triângulo Mineiro. Naturalia 22: 115-129.

Araújo, G. M.; Nunes, J. J.; Rosa, A. G. \& Resende, E. J. 1997a. Estrutura comunitária de vinte áreas de cerrados residuais no município de Uberlândia, MG. Daphne 7: 7-14.

Araújo, G. M.; Guimarães, A. J. M. \& Nakajima, J. N. 1997b. Fitossociologia de um remanescente de mata mesófila semidecídua urbana, Bosque Jonh Kennedy, Araguari, MG, Brasil. Revista Brasileira de Botânica 20: $67-77$.

Carvalho, D. A.; Oliveira-Filho, A. T.; Vilela, E. A. \& Gavilanes, M. L. 1995. Flora arbustivo-arbórea de uma floresta ripária no Alto Rio Grande em Bom Sucesso, MG. Acta Botanica Brasilica 9: 231-245.

Castro, A. A. J. F.; Martins, F. R.; Tamashiro, J. Y. \& Shepherd, G. J. 1999. How rich is the flora of brazilian cerrados ? Annais Missouri Botanical Garden 86: 192-224.

Coutinho, L. M. 1978. O conceito de Cerrado. Revista Brasileira de Botânica 1: 17-23.

Eiten, G. 1972. The cerrado vegetation of Brazil. Botanical Review 38: 201-341.

Embrapa. 1982. Levantamento de reconhecimento de média intensidade dos solos e avaliação da aptidão agrícola das terras do Triângulo Mineiro. Boletim de Pesquisa 1. Serviço Nacional de Levantamento e Conservação de Solos. Rio de Janeiro, RJ.

Felfili, J. M.; Silva-Júnior, M. C.; Resende, A. V.; Machado, J. W. B.; Walter, B. M. T.; Silva, P. E. N. \& Hay, J. D. 1993. Análise comparativa da florística e fitossociologia da vegetação arbórea do cerrado sensu stricto na Chapada Pratinha, DF, Brasil. Acta Botanica Brasilica 6: 27-46.

Gandolfi, S.; Leitão-Filho, H. F. \& Bezerra, C. L. 1995. Levantamento florístico e caráter sucessional das espécies arbustivo-arbóreas de uma floresta mesófila semidecídua no Município de Guarulhos, SP. Revista Brasileira de Biologia 55: 753-767. 
Goodland, R. 1979. Análise ecológica da vegetação do cerrado. In: Goodland, R. \& Ferri, M.G. (Eds.). Ecologia do Cerrado. EDUSP, São Paulo, Itatiaia. Belo Horizonte.

Grombone, M. T.; Bernacci, L. C.; Meira-Neto, J. A. A.; Tamashiro, J. Y. \& Leitão-Filho, H. F. 1990. Estrutura fitossociológica da floresta semidecídua de altitude do Parque Municipal da Grota Funda (Atibaia-Estado de São Paulo). Acta Botanica Brasilica 4: 47-64.

Lopes, A. S. \& Cox, F. R. 1977. Cerrado vegetation in Brazil: an edaphic gradient. Agronomical Journal 69: 828-831.

Mantovani, W. \& Martins, F. R. 1993. Florística do cerrado na reserva biológica de Moji Guaçu, SP. Acta Botanica Brasilica 7: 33-60.

Miranda, I. S. 1993. Estrutura do estrato arbóreo do cerrado amazônico em Alter-do-Chão, Pará, Brasil. Revista Brasileira de Botânica 16: 143-150.

Miranda, I. S. \& Carneiro-Filho, A. 1994. Similaridade florística de algumas savanas amazônicas. Boletim do Museu Paraense Emílio Goelde, Série Botânica 10: $249-267$.

Nascimento, T. M. \& Saddi, N. 1992. Structure and floristic composition in an area of Cerrado in Cuiabá, MT, Brazil. Revista Brasileira de Botânica 15: 4755.

Oliveira-Filho, A. T. \& Machado, J. N. M. 1993. Composição florística de uma floresta semidecídua montana, na Serra de São José, Tiradentes, Minas Gerais. Acta Botanica Brasilica 7: 71-88.

Pedralli, G.; Freitas, V. L. O.; Meyer, S. T.; Teixeira, M. C. B. \& Gonçalves, A. P. S. 1997. Levantamento florístico na Estação Ecológica do Tripuí, Ouro Preto, MG. Acta Botanica Brasilica 11: 191-213.

Ratter, J. A.; Richards, P. W.; Argent, G. \& Gifford, D. R. 1973. Observations on the vegetation of northeastern Mato Grosso 1 . The woody vegetation types of the Xavantina-Cachimbo expedition area. Philosophical Transactions of the Royal Society of London (B) 266: 449-492.

Ratter, J. A.; Askew, G. P.; Montgomery, R. F. \& Gifford, D. R. 1977. Observações adicionais sobre o Cerradão de solos mesotróficos no Brasil Central. In: Ferri, M. (Coord.). IV Simpósio sobre o Cerrado - bases para utilização agropecuária. EDUSP, São Paulo, Ed. Itatiaia, Belo Horizonte.
Ratter, J. A.; Leitão-Filho, H. F.; Argent, G.; Gibbs, P. E.; Semir, J.; Shepherd, G. J. \& Tamashiro, J. Y. 1988. Floristic composition and community structure of a southern cerrado area in Brazil. Notes of the Royal Botanic Garden Edinburgh 45: 137-151.

Ratter, J. A.; Bridgewater, S.; Atkinson, R. \& Ribeiro, J. F. 1996. Analysis of the floristic composition of the brazilian cerrado vegetation II: comparision of the woody vegetation of 98 areas. Edinburgh Journal of Botanic 53: 153-180.

Ribeiro, J. F.; Silva, J. C. S. \& Batmanian, G. J. 1985. Fitossociologia de tipos fisionômicos de cerrado em Planaltina, DF. Revista Brasileira de Botânica 8: 131-142.

Rizzini, C. T. 1963. A flora do Cerrado. In: Ferri, M.G. (Coord). Simpósio sobre o Cerrado. EDUSP, São Paulo.

Rizzini, C. T. 1979. Tratado de Fitogeografia do Brasil - aspectos sociológicos e florísticos. v. 2. Editora HUCITEC / EDUSP. São Paulo, SP.

Rodrigues, R. R.; Morellato, L. P. C.; Joly, C. A. \& Leitão-Filho, H. F. 1989. Estudo florístico e fitossociológico em um gradiente altitudinal de mata estacional mesófila semidecídua, na Serra do Japi, Jundiaí, SP. Revista Brasileira de Botânica 12: 71 84.

Rosa, R.; Lima, S. C. \& Assunção, W. L. 1991. Abordagem preliminar das condições climáticas de Uberlândia (MG). Sociedade \& Natureza 3: 91-108.

Rossi, C. V.; Silva-Júnior, M. C. \& Santos, C. E. N. 1998. Fitossociologia do estrato arbóreo do cerrado (sensu stricto) no Parque Ecológico Norte, BrasíliaDF. Boletin do Herbário Ezechias Paulo Heringer 2: 49-56.

Schiavini, I. \& Araújo, G. M. 1989. Considerações sobre a vegetação da Reserva Ecológica do Panga (Uberlândia). Sociedade \& Natureza 1: 61-66.

Sheperd, G. J. 1994. FITOPAC 1. Manual do usuário. Departamento de Botânica. UNICAMP.

Veloso, P. V. 1992. Sistema fitogeográfico. In: Manual Técnico da Vegetação Brasileira. IBGE. Rio de Janeiro, RJ.

Vilela, E. A.; Oliveira-Filho, A. T.; Carvalho, D. A. \& Gavilanes, M. L. 1995. Flora arbustivo-arbórea de um fragmento de mata ciliar no Alto Rio Grande, Itutinga, Minas Gerais. Acta Botanica Brasilica 9: 87-100. 